\title{
Transoesophageal echocardiography improves the diagnostic value of cardiac ultrasound in patients with carcinoid heart disease
}

\author{
Lennart Lundin, Johan Landelius, Bertil Andrén, Kjell Öberg
}

\begin{abstract}
Transthoracic and transoesophageal cardiac echocardiography and Doppler investigations were performed in 31 consecutive patients with malignant midgut carcinoid tumours. The transoesophageal images allowed measurement of the thickness of the atrioventricular valve leaflets and the superficial wall layers on the cavity side of both atria. The mean thickness of the anterior tricuspid leaflet was significantly greater than that of the mitral valve-a difference not seen in a control group of age-matched patients without carcinoid tumours and with normal cardiac ultrasound findings. In addition, the edges of the tricuspid leaflets were thickened giving them a clubbed appearance. Tricuspid incompetence was detected transoesophageally in $71 \%$ of the patients with carcinoid compared with $57 \%$ by transthoracic investigation. The inner layer of the right atrial wall in the carcinoid patients was significantly thicker than that of the left atrium and that of both atria in the controls. Furthermore, patients with other signs of severe carcinoid heart disease had significantly thicker mean right atrial luminal wall layer than those with less or no signs of right heart disease.

Transoesophageal cardiac ultrasound investigation improved the diagnosic accuracy and seemed to show the structural changes typical of carcinoid heart disease established by histopathological investigations.
\end{abstract}

Department of Cardiology,

University Hospital

Uppsala, Sweden

L Lundin

Department of Clinical Physiology,

University Hospital

Uppsala, Sweden

J Landelius

B Andrén

Ludwig Institute of Cancer Research, University Hospital, Uppsala, Sweden K Oberg

Correspondence to Dr Lennart Lundin Department of Cardiology, Medical Clinic, University Hospital, S-751 85 Uppsala, Sweden.

Accepted for publication 9 March 1990

Malignant mid-gut carcinoid tumours are often associated with a characteristic heart disease with pathognomonic endocardial deposition of myofibromatous tissue. ${ }^{1-3}$ In most cases these lesions are confined to the right heart where they are localised in the subendocardium of both cavities and on the tricuspid and pulmonary valves. The cusps are thickened and immobilised resulting in stenosis or incompetence of the valves or both. These features cause pressure and volume overload of the right side of the heart. Restrictive right heart disease has also been described and this is probably the result of the carcinoid related endocardial fibrous thickening of the heart chamber walls. ${ }^{45}$ In several patients with severe disease similar changes were found in the left heart at necropsy-in some cases associated with cardiac shunts or bronchial carcinoids. ${ }^{167}$

At present transthoracic echocardiography including imaging and Doppler investigations seems to be the most useful and suitable diagnostic tool for detecting carcinoid heart disease. ${ }^{8-10}$ The most commonly described echocardiographic findings are abnormalities of the morphology and motion of the tricuspid and pulmonary valves and an increase in the dimensions of the right heart cavities. In severe cases of right ventricular overload, paradoxical interventricular septal contractions towards the right ventricle have been reported. Doppler ultrasound investigation often detects abnormal valvar blood flow patterns, the most common finding being tricuspid incompetence.

Transoesophageal echocardiography contributes important information to cardiac investigations in aortic and mitral valve disorders. ${ }^{11}$ Transoesophageal echocardiography also aids the detection of atrial abnormalities and is a valuable technique for perioperative and intraoperative monitoring of myocardial function. ${ }^{1213}$ The close proximity to the heart facilitates the use of high frequency transducers and reduces common causes of artefacts, both factors that improve the image resolution, especially of the base of the heart.

We examined patients with carcinoid tumours prospectively by both transthoracic and transoesophageal echocardiography to determine whether the transoesophageal approach give additional diagnostic information.

\section{Patients and methods}

\section{PATIENTS}

Transthoracic and transoesophageal investigations were attempted in 38 consecutive patients with malignant mid-gut carcinoid tumours. Seven patients were excluded from further analyses because of technically inadequate images in five patients and difficulties in advancing the transoesophageal probe and the inability of two patients to tolerate the probe. The remaining group comprised 12 women and 19 men (mean age 58 years (range 41-75)). Two years before one patient had successfully been given tricuspid and pulmonary valve replacements because of severe carcinoid heart disease and right heart failure. The diagnosis of carcinoid disease was histologically confirmed in all patients by the 
Grimelius and Masson silver staining techniques and by immunohistochemical examination of tumour tissue obtained at biopsy or operation. ${ }^{14}$ Abdominal computed tomography and ultrasound showed liver metastases in all patients and all had the classic signs and symptoms of carcinoid syndrome-that is flush, diarrhoea, and raised urinary excretion of 5-hydroxyindoleacetic acid.

Thirteen patients (eight men and five women, mean age 48 years (range 28-76)) investigated by transoesophageal cardiac ultrasound because cardiac disease was suspected served as a control group for the transoesophageal measurements of valve cusp thickness and atrial wall dimension. In these 13 individuals the findings at transthoracic and transoesophageal echocardiography were regarded as normal.

\section{METHODS}

All ultrasound investigations were performed with a Hewlett-Packard real-time scanner (HP Sonos 500, version $\mathrm{K}$ ). The transthoracic images were almost exclusively obtained with a $2.5 \mathrm{MHz}$ imaging probe (sometimes 3.5 $\mathrm{MHz}$ ) that also provided facilities for pulsed wave Doppler including colour flow mapping. The methods we used for imaging and measuring are described elsewhere. ${ }^{10}$ The tricuspid valve was regarded as abnormal if the leaflets were thickened and cusp motion was reduced. When there was also concomitant pansystolic valvar incompetence (see below) structural abnormalities were actively sought. All patients were examined by pulsed and continuous wave Doppler recordings with spectral analyses and colour flow mapping. A 2.5 MHz imaging transducer was used for the colour flow mapping in all patients because this frequency is more sensitive in adult patients than higher frequencies. Though Doppler ultrasound in several investigations of healthy individuals showed valvar incompetence, especially of the tricuspid valve,

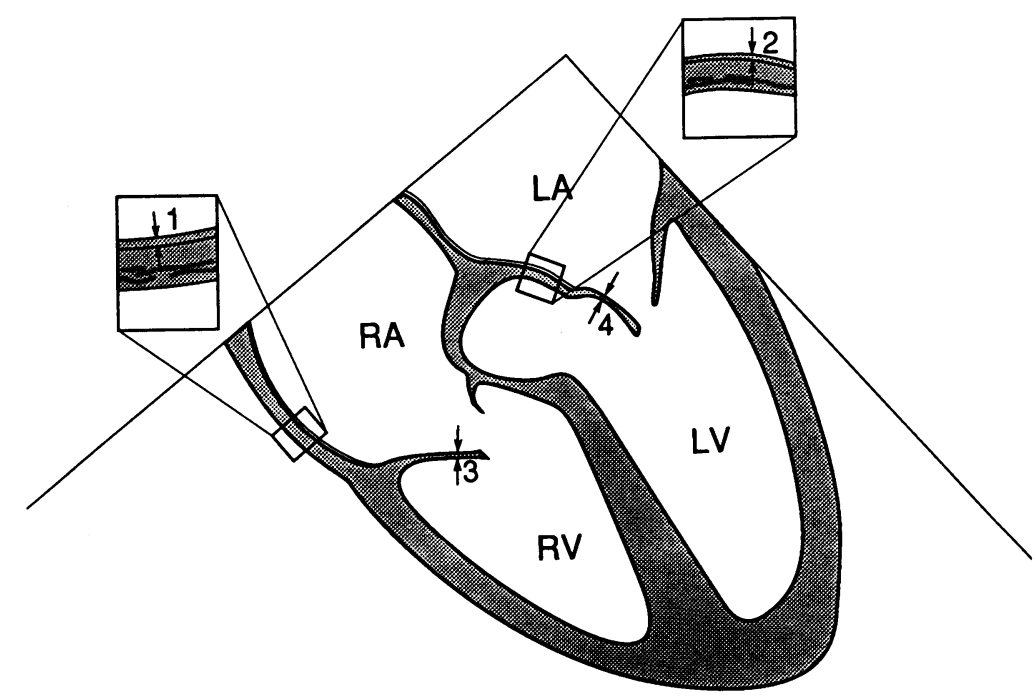

Figure 1 Schematic illustration of the measurements in the transoesophageal images: superficial wall layer on the cavity side of the right (1) and left (2) atrium and thickness of middle part of the anterior leaflet of the tricuspid (3) and mitral (4) valves. $L A$, left atrium; $L V$, left ventricle; $R A$, right atrium; $R V$, right ventricle. there is no definition of what constitutes an abnormal degree of regurgitation detected by Doppler. Recordings showing pansystolic tricuspid valve regurgitation and pandiastolic pulmonary valve regurgitation.were regarded as abnormal in this study.

Transoesophageal echocardiography was performed with a Hewlett-Packard echoscope consisting of a $5.0 \mathrm{MHz}, 64$ element, $11 \times 14 \mathrm{~mm}$ transducer mounted on the distal tip of a gastroscope. The distal part of the echoscope could be manipulated by two external wheels with a deflection range of $90^{\circ}$ left/ $90^{\circ}$ right and $120^{\circ}$ up (anterior) $/ 90^{\circ}$ down (posterior). Patients fasted for 4 hours before the procedure about which they were fully. informed. The echoscope was introduced with the patient in the supine, left lateral position after the echoscope tip had been covered with anaesthetic gel and slight pharyngeal aerosol anaesthesia had been applied. We recorded all registrations on a VHS video recorder using the PAL/Secam standard.

The Hewlett-Packard system displays distance measurements by means of internal callipers on the screen to an accuracy of one hundredth of a $\mathrm{mm}$ on images when the closest ranges of the system are used. This numerical accuracy is based on the physical properties of the internal digital matrix of the scanner. Such an accuracy is spurious compared with the capabilities of other components of the imaging system. The best image resolution obtainable is that of the wavelength of the emitted ultrasound at the velocity of speed of $1570 \mathrm{~m} / \mathrm{s}$. Thus the resolution would be $0.3 \mathrm{~mm}$ in the axial direction of the sector image. But other factors also influence resolution-such as, transducer sensitivity and the engineering physics used to transform the returning analogue reflection pattern for conversion into a digital scan matrix and the different algorithms used to produce the final image presented by a video system. In practical terms, if all measurements on the transoesophageal images are optimised along the axial direction of the image it is reasonable to claim a precision of $0.5 \mathrm{~mm}$ because there are no image artefacts and the transducer is close to the heart. Because the technique is used to measure relative differences between pairs of structures the precision of the true physical measurement is less important.

We measured the thickness of the anterior tricuspid and mitral leaflets at their midpoints and the thickness of a layer on the cavity sides of the right and left atrial wall (fig 1). Two experienced investigators independently selected the best available images to measure these dimensions from the video recordings. The results, taken from the system as they were presented, were compared by means of Student's $t$ test for paired values. No significant interobserver difference was found. The mean values of the two observers were thereafter corrected to the nearest $0.5 \mathrm{~mm}$.

The data were assessed by Student's $t$ test for paired values and independent groups and by analysis of variance with multiple comparisons when appropriate. 
Table 1 Transthoracic Doppler and echocardiographic findings in 31 patients with malignant mid-gut carcinoid tumours

\begin{tabular}{lrc}
\hline Finding & No. & $\%$ \\
\hline Tricuspid abnormalities: & $19 / 31$ & 61 \\
Thickened cusp & $8 / 30$ & 26 \\
Decreased cusp motion & $6 / 30$ & 20 \\
Tricuspid incompetence & $17 / 30$ & 57 \\
Pulmonary valve incompetence & $9 / 26$ & 35 \\
Right atrial enlargement & $18 / 31$ & 58 \\
Right ventricular enlargement & $10 / 31$ & 32 \\
Paradoxical septal contractions & $8 / 31$ & 26 \\
Normal right heart & $7 / 31$ & 23 \\
\hline
\end{tabular}

\section{Results}

Transthoracic investigation

A right heart abnormality was found in 24 $(77 \%)$ of the 31 patients. Table 1 shows the frequency of the different abnormalities. Tricuspid abnormalities, including the detection of pansystolic tricuspid incompetence by Doppler, were found in $19(61 \%)$. The tricuspid leaflets were thickened in eight $(26 \%)$ and in severe cases the edges were clubbed. The image resolution did, however, not permit the thickness of the tricuspid leaflet to be measured. Nine $(35 \%)$ of the 26 patients in whom Doppler registrations of the pulmonary flow were satisfactory had pandiastolic pulmonary valve incompetence. The right atrium and ventricle were enlarged in $18(58 \%)$ and 10 $(32 \%)$. respectively, and eight $(26 \%)$ of the patients had paradoxical interventricular septal contractions.

In seven patients transthoracic echocardiography showed a normal right heart (group 1). In eight it showed morphological or functional abnormalities of the tricuspid valve, enlargement of both the right atrium and ventricle, and paradoxical septal systolic contractions (group 3 ). The remaining 16 patients (group 2) had one or more but not all the signs of right heart disease seen in the patients in group 3.

\section{Transoesophageal investigation}

Transoesophageal images of the four cavities of the heart and the aortic, mitral, and tricuspid valves were adequate in all patients but the pulmonary valve was visualised only in a few.

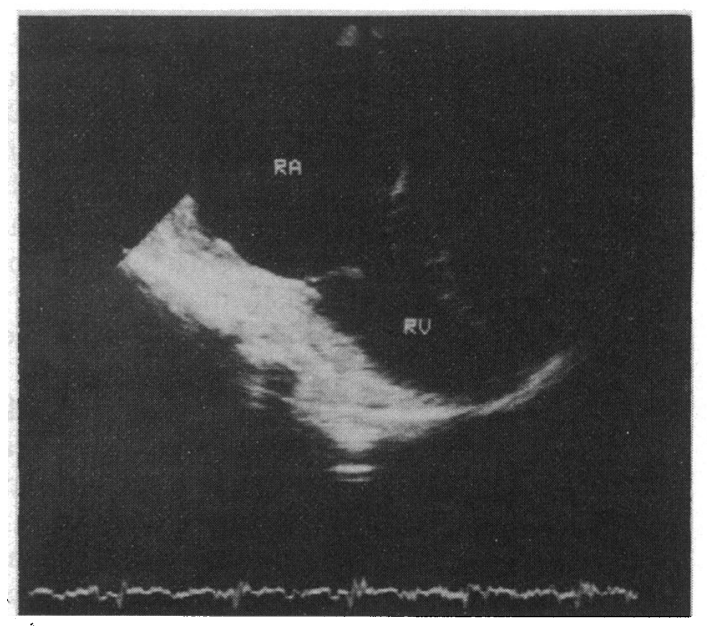

Figure 2 Transoesophageal cross sectional echocardiogram from a patient with carcinoid hear disease showing abnormal anterior tricuspid leaflet with a clubbed edge to the cusp. $R A$, right atrium; $R V$, right ventricle.

There were similar difficulties in obtaining adequate colour Doppler registrations of the pulmonary flow; colour flow mapping of the other three valves was good.

In 16 patients ( $53 \%$ ) thickening of the edges of the tricuspid leaflets gave them a clubbed appearance (fig 2). Some patients in groups 1 and 2 but none of the controls had clubbed tricuspid leaflets. All patients in group 3 with native valves had thickened leaflets and incompetent tricuspid valves. In one patient who 2 years earlier had tricuspid and pulmonary valve replacements, the bioprosthetic valves were competent and there were no morphological abnormalities. Tricuspid incompetence was seen in two patients in group 1 and 13 in group 2 (table 2). In total, $22(73 \%)$ of the patients with carcinoid had tricuspid incompetence. Tricuspid incompetence was detected by transoesophageal colour flow mapping in five patients in whom transthoracic examinations by pulsed and continuous wave Doppler and colour flow mapping had not shown an incompetent valve.

The mean thickness of the tricuspid leaflet was significantly greater in group 3 than in group $1(p<0.01)$, group $2(p<0.05)$, or the

Table 2 Transoesophageal ultrasound findings in 31 patients with malignant mid-gut carcinoid tumours grouped by degree of carcinoid heart disease and in 13 controls with normal cardiac ultrasound findings and without carcinoid tumours

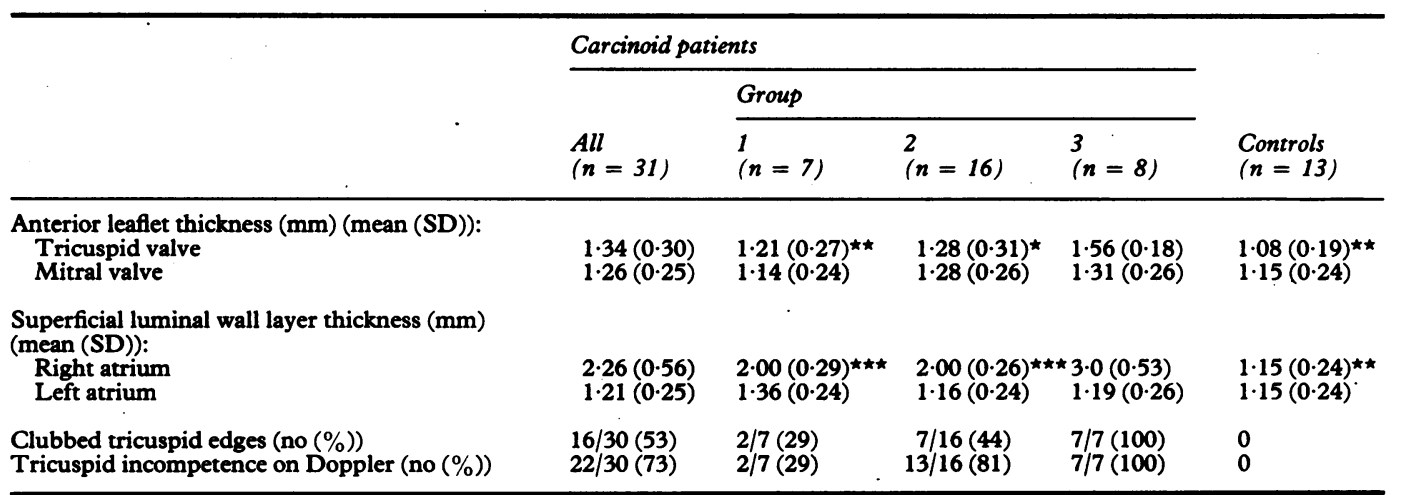

Asterisks indicate significant differences when group 3 was compared with groups 1, 2, and controls.

${ }^{\star} \mathrm{p}<0.05 ;{ }^{\star \star} \mathrm{p}<0.01 ;{ }^{\star \star \star} \mathrm{p}<0.001$. 
Figure 3

Transoesophageal cross sectional echocardiogram in a patient with carcinoid heart disease $(A)$ and in a patient with normal cardiac ultrasound findings without carcinoid tumour (B). The images show thickening of the superficial wall layer on atrium in the patient with carcinoid disease. $L A$, left atrium; $R A$, right atrium, $R V$, right ventricle. The scale is the same in both figures. the cavity side of the right


control group ( $p<0.001)$. There were no significant differences in leaflet thickness between the controls and groups 1 and 2 (table 2). Mitral leaflet thickness did not differ significantly between any of the four groups. There was no significant difference between the tricuspid and mitral cusp thickness in any of the groups.

The mean thickness of the normally rather faint wall layer (fig 3B) on the cavity surface of the right atrium was significantly greater $(p<0.001)$ than that of the left atrium in the 31 patients with carcinoid; there was no significant difference between these dimensions in the control group (table 2). In addition, in $90 \%$ of the patients with carcinoid disease this wall layer was $>+2 \mathrm{SD}$ thicker than the mean for

\footnotetext{
Figure 4

Transoesophageal cross sectional echocardiogram from a patient $v$ ith carcinoid heart disease showing irregular luminal margin of the right atrial wall (indicated). $R A$, right atrium; $R \dot{V}$, right ventricle.
}

the control patients. The patients in group 3, who had the most advanced carcinoid heart disease at the transthoracic echocardiographic examination, also had a significantly thicker $(p<0.001)$ right atrial wall layer than the patients in groups 1 and 2 or the controls. In addition, the luminal margin of the right atrial wall was often more echodense in patients with carcinoid heart disease, giving the wall a "typical" double contour (fig 3A). In some patients the inner margin of the atrial wall formed crypts and ridges (fig 4). The cardiac abnormalities detected by ultrasound examination were subsequently confirmed at operation when two patients with severe carcinoid heart disease had valve replacement.

\section{Discussion}

In this prospective study of patients with malignant mid-gut carcinoid tumours, transthoracic cardiac ultrasound investigation detected right heart abnormalities that resembled those reported earlier, although they were somewhat more common in the present study. ${ }^{18-10}$ The right heart was abnormal in $77 \%$ (22/30); the most common abnormalities were morphological or functional changes in the tricuspid valve and enlargement of the right atrium. Pulmonary valve incompetence, right ventricular enlargement, and paradoxical systolic septal contractions were found in $26-35 \%$ of the patients.

Transoesophageal cardiac ultrasound gave a closer view of the shape and function of the atrioventricular valve and the characteristics of the atrial walls. We often found that the leaflet margins were clubbed and the cusps of the tricuspid valve were thickened; this accords with histopathological findings in patients with carcinoid disease. ${ }^{1-3}$ We found that the middle of the anterior tricuspid leaflet was thicker in patients with severe carcinoid disease than in patients with less severe disease and in the controls. The echocardiographic measurements in carcinoid patients and in the controls were somewhat higher than Sahaskul et al found in necropsy specimens of normal hearts. ${ }^{15}$ The reason for this discrepancy is not obvious but angular mismatch between the ultrasonic beam direction and the measured structure could result in an overestimation by the ultrasonic measurements. We were, however, careful to choose images obtained with the beam at $90^{\circ}$ to the structure. The discrepancy could be the result of the technical difficulties with echocardiographic resolution described in the methods section. However, the limit of resolution is likely to be $<0.5 \mathrm{~mm}$ rather than $>0.5 \mathrm{~mm}$. Measurements made with a micrometer might be underestimations because the tissues may have been compressed. Finally, living tissues with normal tissue fluid dynamics may truly have different dimensions after death.

Although the angle between the tricuspid flow and the ultrasound beam often made flow velocity determinations by spectral Doppler analysis impossible, transoesophageal colour 
flow mapping detected tricuspid incompetence in five patients in whom it was not detected by transthoracic investigations. The pulmonary valve and its flow patterns, however, were more often successfully investigated by transthoracic rather than transoesophageal investigation, although in a few patients the pulmonary valve characteristics were well displayed by transoesophageal echocardiography.

Because the transoesophageal transducer was close to the atria it generated images that showed different types of atrial wall morphology in patients with and without carcinoid heart disease. In controls the walls of both atria had a double contour with a thinner echodense layer of similar thickness on the cavity side. In most patients with carcinoid heart disease this layer was sharper and significantly thicker in the right atrium than in the left atrium and thicker than in both atria in the controls. The abnormalities of the right atrial wall were also more pronounced in patients with other signs of severe carcinoid heart disease. The mean (SD) luminal atrial wall layer which was $2 \cdot 26$ $(0.56) \mathrm{mm}$ in the carcinoid patients, could be the echocardiographic image of the carcinoid endocardial myofibromatous tissue deposition, which histology showed was approximately $2 \mathrm{~mm}$ thick. ${ }^{3}$ At operation in two patients with severe carcinoid heart disease we found patchy irregularities that seemed to increase the endocardial thickness. This may explain why transoesophageal echocardiography showed thickening of the inner layer of the right atrial wall and irregularities of the inner surface of the atrial wall which in some sites may have made the atrial wall trabeculation more pronounced.

We found that transoesophageal echocardiography improved the sensitivity of cardiac ultrasound investigation in patients with carcinoid tumours by showing the subendocardial myofibromatous tissue that is typical of carcinoid heart disease. The increase in tissue dimensions accorded with earlier histological estimations. Transthoracic cardiac ultrasound currently seems to be a suitable method of screening patients with carcinoid disease for cardiac abnormalities. The high resolution images obtained by the transoesophageal technique offer a possibility for detection and follow up of less advanced morphological and functional abnormalities. The place of transoesophageal echocardiography in routine practice, however, remains uncertain except in those patients who are poor subjects for transthoracic echocardiography. In patients with prosthetic valves the technique might detect carcinoid lesions on the prosthetic valves; such lesions have been found at necropsy in one patient. ${ }^{16}$

This study was supported by the Faculty of Medicine, University of Uppsala, the Ludwig Institute for Cancer Research, University Hospital, Uppsala, and the Swedish Nationa Association Against Heart and Chest Diseases.

1 Roberts WC. Sjoerdsma A. The cardiac disease associated with the carcinoid syndrome. Am JMed 1964;36:5-34.

2 Trell E, Rausing A, Ripa J, Torp A, Waldenström J Carcinoid heart disease. Clinicopathologic findings and follow-up in 11 cases. Am J Med 1973;54:433-44.

3 Ferrans VJ, Roberts WC. The carcinoid endocardial plaque. An ultrastructural study. Hum Pathol 1976;7:387-408.

4 Roberts WC, Mason DT, Wright LD. The nondistensible right atrium of carcinoid of the heart. Am J Pathol right atrium of
$1965 ; 44: 627-31$

5 McGuire MR, Pugh DM, Dunn MI. Carcinoid heart disease. Restrictive cardiomyopathy as a late complicadisease. Restrictive cardiomyopathy
tion. J Kans Med Soc 1978;79:661-2.

6 Von Bernheimer H, Ehringer H, Heistracher P, et al. Biologisch aktives, nicht metastasierendes Bronchuscarcinoid mit Linkshertz-syndrom. Wien Klin Wochenschr 1960;72:867.

7 Schweizer W, Gloor F, von Bertrab R, Dubach UC. Carcinoid heart disease with left sided lesions. Circulation 1964;24:253-7.

8 Callahan JA, Wroblewski EM, Reeder GS, Edwards WD, Seward JB, Tajik AJ. Echocardiographic features of carcinoid heart disease. Am J Cardiol 1982;50:762-8.

9 Howard RJ, Drobac M, Rider WD, et al. Carcinoid heart disease: diagnosis by two-dimensional echocardiography Circulation 1982;66:1059-65.

10 Lundin L, Norheim I, Landelius J, Oberg K, TheodorssonNorheim E. Carcinoid heart disease: relationship of circulatin E. Carcinoid heart disease: relationship of circulating vasoactive substances to ultrasound-detec
cardiac abnormalities. Circulation 1988;77:264-9.

11 Erbel R, Rohmann S, Drexler M, et al. Improved diagnostic value of echocardiography in patients with infective endocarditis by transesophageal approach. A prospective study. Eur Heart J 1988;9:43-53.

12 Aschenberg W, Schlüter M, Kremer P, Schröder E, Siglow $V$, Bleifeld W. Transesophageal two-dimensional echocardiography for the detection of left atrial appendage thrombus. J Am Coll Cardiol 1986;7:163-6.

13 Shunei K, Shinichi T, Makoto M, et al. Immediate and early postoperative evaluation of results of cardiac surgery by transesophageal two-dimensional echocardiograph. culation 1987;76(5Pt2):(suppl V): 113-21.

14 Grimelius L, Wilader E. Silver stains in the study of endocrine cells of the gut and pancreas. Invest Cell Patho 1980;3:3-6.

15 Sahaskul Y, Edwards WD, Naessens JM, Tajik AJ. Age related changes in aortic and mitral valve thickness: related changes in aortic and mitral valve thickness: implications for two-dimensional echocardiography based on autopsy study of 200

16 Schoen FJ, Hausner RJ, Howell JF, Beazely HL, Titus JL. Porcine heterograft valve replacement in carcinoid hear disease. J Thorac Cardiovasc Surg 1981;81:100-5. 